\title{
Plastic and Reconstructive Surgery
}

\section{COSMETIC SURGERY I}
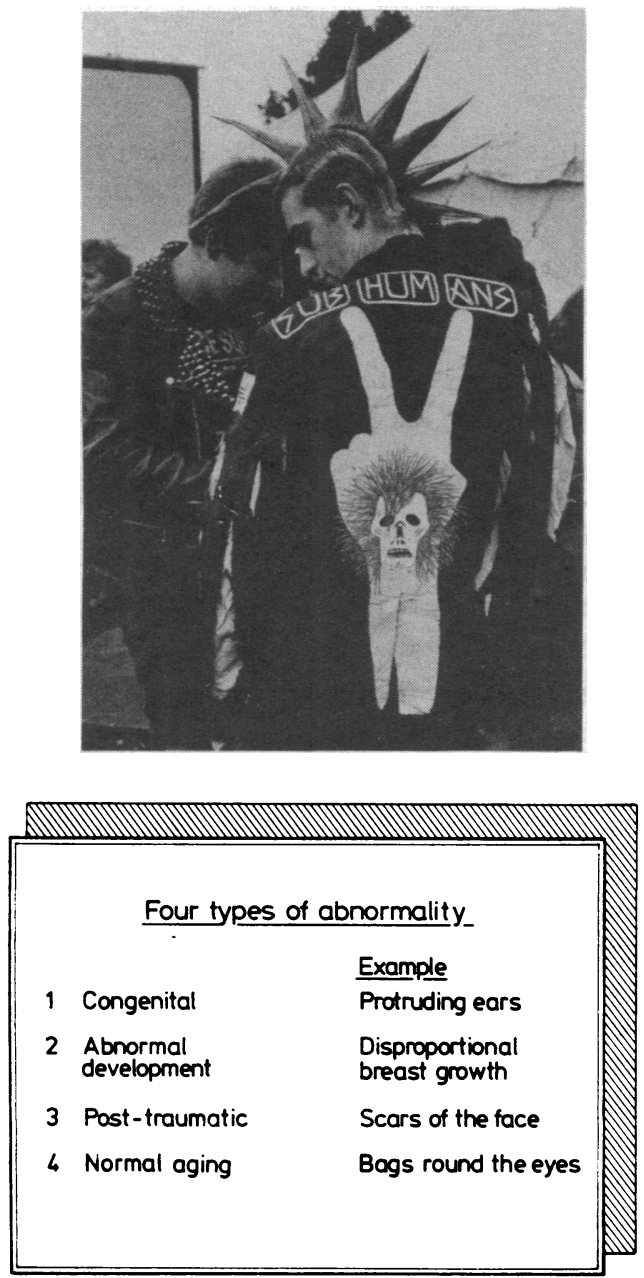

Display is an important animal instinct, which may manifest itself in primitive societies by body adornment with various dyes or elaborate scars and even more drastic anatomical changes such as increasing the size of the lips or the lobes of the ears. Our own society's equivalents include tattooing, the wearing of makeup and jewellery, and the changing of hair styles. This may be reinforced for many of us by the wish to conform with our peers or with the demands of society. It seems that in all of us there are behavioural patterns or instincts to alter or promote our appearance.

Recently a concept of aestheticality has been introduced: just as some people are musical, so others have a highly developed sense of body image and thus are more motivated to have any abnormality in their appearance corrected. Abnormalities may be produced in one of four ways (table). Whichever way an abnormality arises, many people can compensate satisfactorily. Others, however, undergo immense psychological distress as a result of their perceived appearance, and this may be exacerbated by teasing at school or at work or by the pressures of contemporary advertising, which equates youth and beauty with money, success, and happiness.

The reason why some people request cosmetic surgery when others with an apparently identical problem do not is, however, difficult to understand for cosmetic surgery is often sought by attractive people with slight imperfections as well as by people with gross abnormalities. Detailed psychological assessments of patients requesting cosmetic surgery have shown many to be at the hysterical end of the spectrum of normal personalities. This does not mean that patients who fall into this group should not be treated by cosmetic surgery for often this may work out more satisfactory and cheaper than treating them with drugs or psychotherapy over a long period. Indeed, cosmetic surgeons have been called psychiatrists who treat patients with a scalpel rather than with drugs.

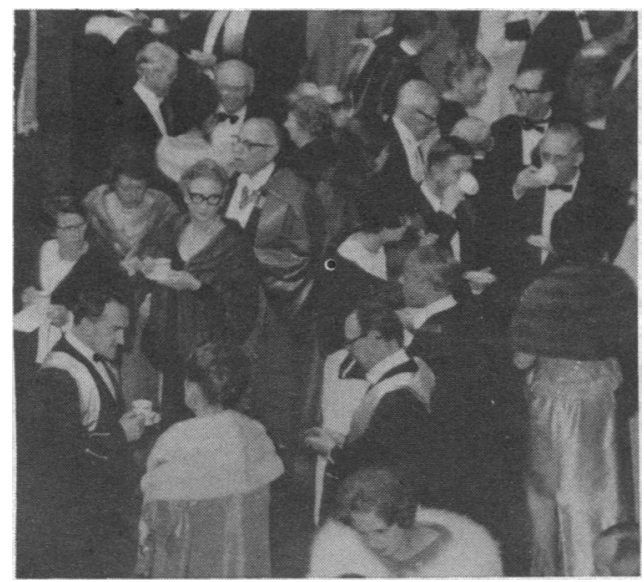

For many cosmetic surgery may be an acceptable treatment for overcoming a personal crisis, and without doubt such patients if properly referred by a sympathetic general practitioner may be helped by cosmetic surgery. Unfortunately, some patients think they cannot get a sympathetic hearing from their general practitioner and therefore refer themselves directly to a plastic surgeon or to a financially motivated clinic that advertises. Cosmetic surgery is no longer only for the rich and famous or the obsessive and neurotic. It is a service demanded by the public and is justifiable on social, psychological, and maybe even economic grounds and should therefore be available through the National Health Service as well as privately.

There is one type of patient to avoid operating on. This is the dysmorphophobic, who is psychotic and becomes besotted with one part of his or her body and can never be satisfied no matter how excellent the Conforming display. surgery undertaken. These patients can become extremely disturbed. 


\section{Cosmetic surgery of the aging face}
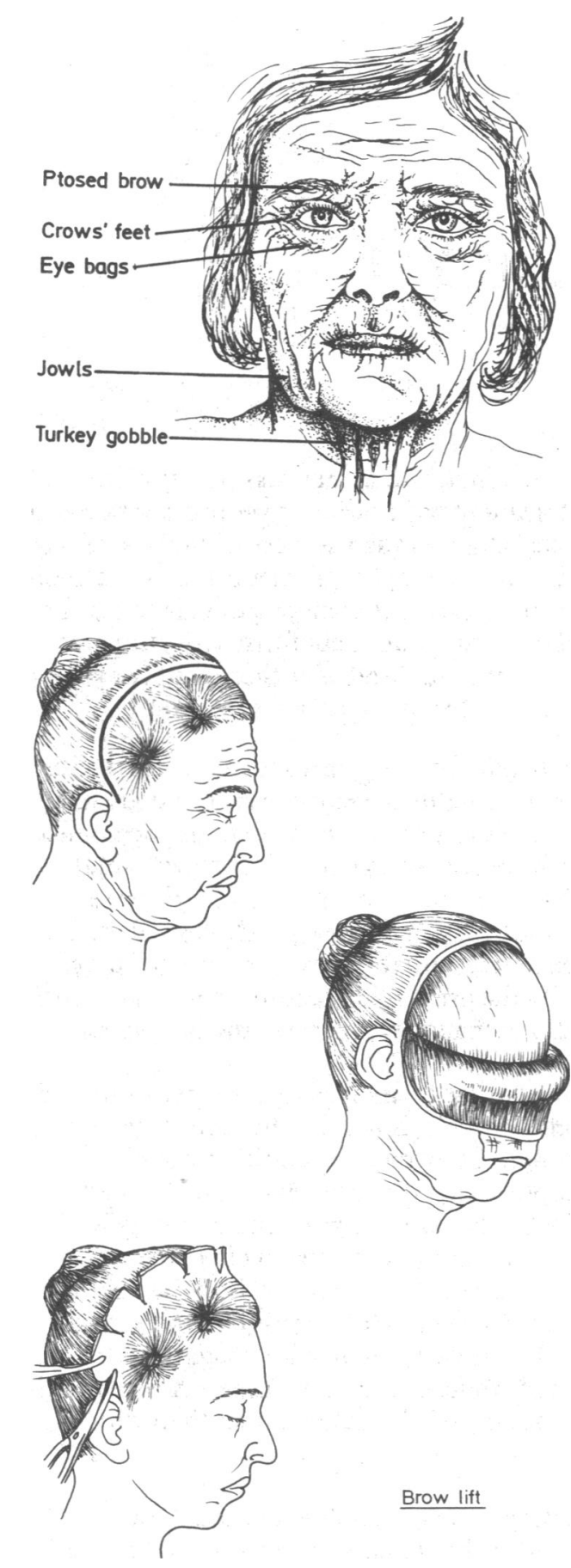

All structures in the body, including the skin and underlying facial muscles, age. The epidermis becomes flatter with a decreased turnover of cells. The major changes are in the dermis, which becomes thinner because of the loss of collagen and fluid and where, in addition, a decrease in the cross linkage of the collagen fibres is accompanied by a loss of elastin fibres. Thus the skin develops a fixed laxity rather than the reversible extensibility of youth. Muscle weakness results in wrinkles and deep creases at the points of greatest mobility-that is, around the eyes and mouth. This, together with the normal centripetal redistribution of fat, results in prominent accumulations around the eyelids, because of weakness of the obicularis muscle, and around the angle of the jaw, forming jowls, because of the weakness in the platysma.

\section{Blepharoplasty}

The eyes are important in communication and are said to reflect a person's inner being. The essential features of the aging process that affect them are:

(1) Ptosis of the eyebrows due to a weak frontalis muscle. Women disguise this by plucking hair from the lower part of the eyebrow and reinforcing the upper part with a pencil.

(2) Excess skin, particularly in the upper eyelid. When the brow is raised this may be less apparent. To a lesser extent excess skin may also develop in the lower eyelid.

(3) Herniation of fat, through the weak obicularis muscle, producing bags, particularly in the medial canthus of the upper lid and in the whole of the lower lid. There may be a familial predisposition to forming baggy eyelids, and this is usually more apparent early in the day. In younger people bagginess may be produced in the lower eyelids by hypertrophy of the obicularis muscle, and in these cases there may not be an excess accumulation of fat.

(4) The development of creases ("crows' feet") radiating out from the lateral canthus.

Before undertaking surgery the physical problem must be diagnosed exactly. Important medical causes of oedema of the eyelids should be eliminated, visual acuity assessed, and the presence of normal tear secretion and drainage checked. In older patients laxity in the lower lid margin with early ectropion should be looked for with care. Both lifting of the brow and reduction of the eyelids may be done under local anaesthesia with sedation on a day case principle or under general anaesthesia.

Brow lift is done through a coronal incision set well back in the hair and undermining down to the supraorbital rim. The forehead is then pulled upwards, taking care to preserve the cutaneous nerves and excising the procerus muscle overlying the glabella area of the nose. The scalp is sutured after excision of the appropriate amount. In general, the procedure is free from complications (apart from the development in some cases of an anaesthetic area in the central forehead). It can appreciably improve the appearance of the eyes.

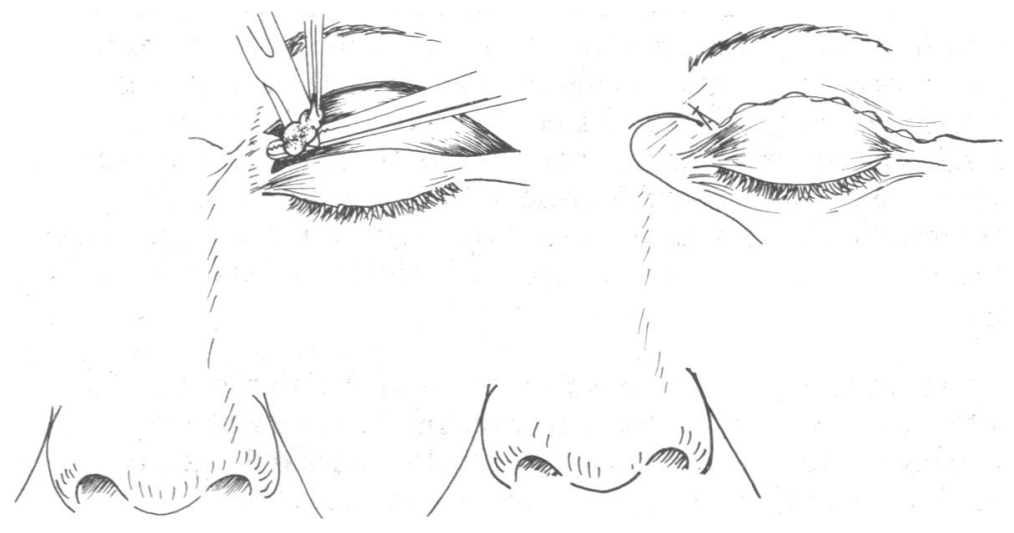

Upper eyelid reduction is performed by excising an ellipse of skin from the upper lid together with excess fat from the medial canthal region-a procedure free from complications provided care is taken. 

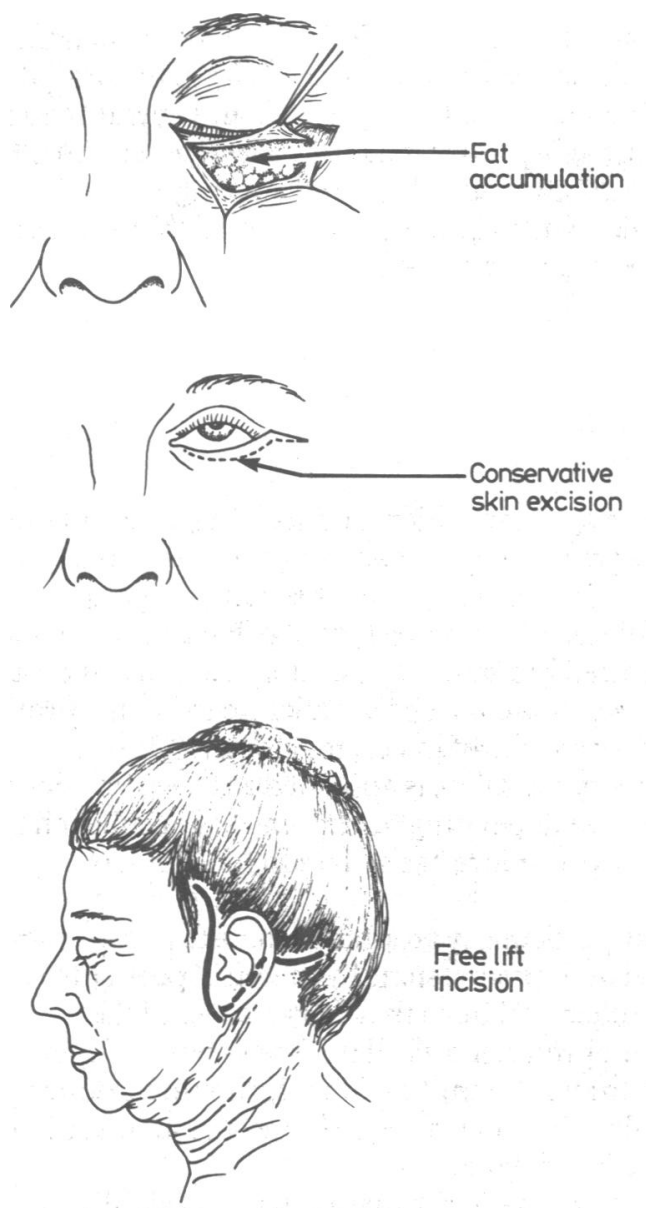

Free lift incision
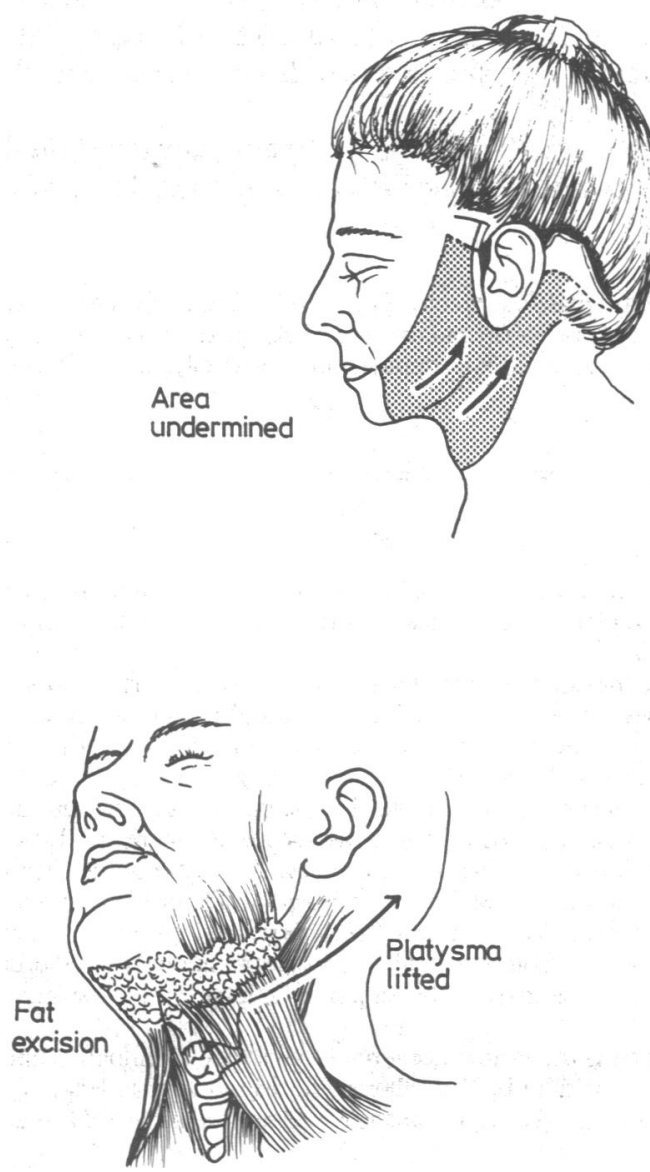

Lower eyelid reduction is done through an incision along the tarsal plate just below the eyelashes and extending out laterally into the crows' feet. Excess fat causing the bags is removed as, at the same time, is any excess skin. This particular procedure requires extreme skill as it is a delicate balance between excising enough skin to produce the desired cosmetic improvement and not producing an ectropion. Many patients who have had the right amount of skin excised initially have a minimal ectropion, often due to postoperative oedema, which settles within a week. Occasionally too much fat may be excised producing a rather cachectic look, and this obviously requires judgment. The ultimate complication of blindness occurs in one in 500000 cases. It is caused by a retrobulbar haematoma, which if diagnosed early can be relieved. The fine creases are not altered, but it is a good procedure for dealing with bags in the lower lids.

\section{Rhytidectomy}

With weakening of the platysma muscle the support to the neck and chin region is lost. Fat becomes redistributed in the face causing jowls to develop in the cervicomandibular area and, in the more obese, a submental collection of fat. The platysma muscle can also form into folds under the chin, producing a so called turkey gobble.

In the standard face lift operation a preauricular incision extending around the back of the ear is continued perpendicularly into the posterior scalp at a height well covered by hair. The skin is then mobilised as a large flap via this incision forwards almost to the nasolabial fold and downwards towards the midline of the neck in a plane superficial to the platysma muscle to avoid damaging the facial nerve. In some patients a submental incision is required to facilitate excision of fat in this area. Any excess fat lying superficial to the platysma muscle can be excised with obvious benefit to the jowls. At the same time the platysma muscle is repositioned by redraping its posterior border and hitching it up high onto the sternomastoid muscles just under the ear. This recreates a sharper angle at the junction of the neck and submental area. Not all patients, however, require this surgery to the platysma.

In general, the standard face lift does little for the mid-face and nothing for the creases in the upper lip. Its major indication is for the neck region, where gratifying results can be achieved. Smaller procedures such as "minilifts" may produce an initial improvement, but experience has shown that these are not sustained. As described it is not a minor procedure, and I think it as best done under general anaesthesia with a stay of two to three days in hospital.

As for any surgery, there are complications. Three per cent of patients have large haematomas requiring drainage, and roughly $15 \%$ have small haematomas, which resolve spontaneously. Between one and five per cent of patients sustain sensory nerve injury, most commonly to the greater auricular nerve supplying the lower part of the ear. More importantly, in up to $2 \%$ of cases the facial nerve may be damaged. This is usually a neuropraxia, and the most common branches injured are the cervicomandibular branch and the branch to the frontalis. Scars in front of the ear heal well. The scar extending horizontally behind the ear sustains the main brunt of tension in a face lift and often has areas of minor necrosis and quite obvious hatch marks, which are produced by the skin sutures. Fortunately this scar should be covered by normal hair growth provided it is positioned high enough in the scalp.

This operation has now become far more common for men. In general, the results are less good than in women, and a higher motivation is required. The scars can be covered in front of the ears with lengthening side boards, but the growth of the beard behind the ears can be a problem. 


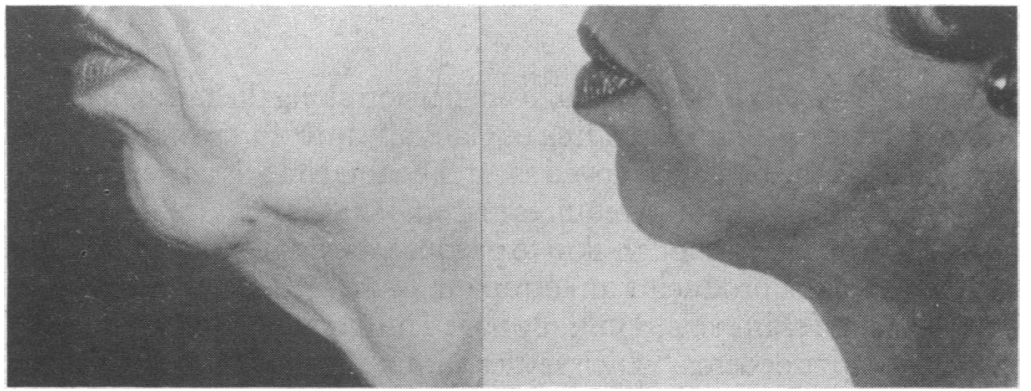

I am often asked, "How long does the benefit from these procedures last?" Within the limitations of personal variation a face lift and eyelid reduction set the aging process back between seven and 10 years. Unfortunately, we all go on aging, and surgery will not alter the rate at which this occurs; it merely sets the clock back.

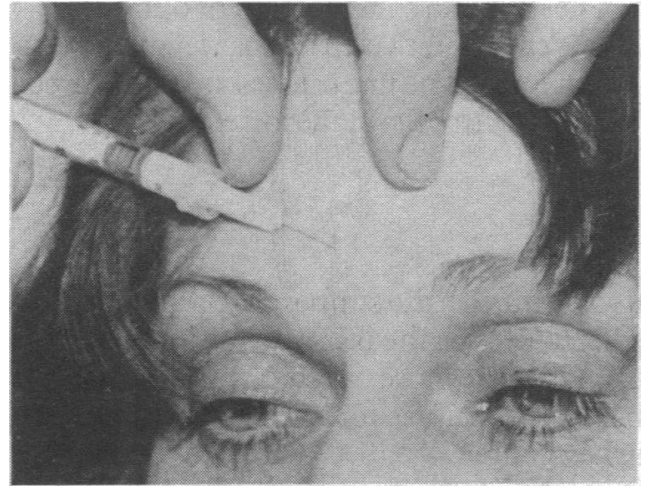

Collagen injection of crease.
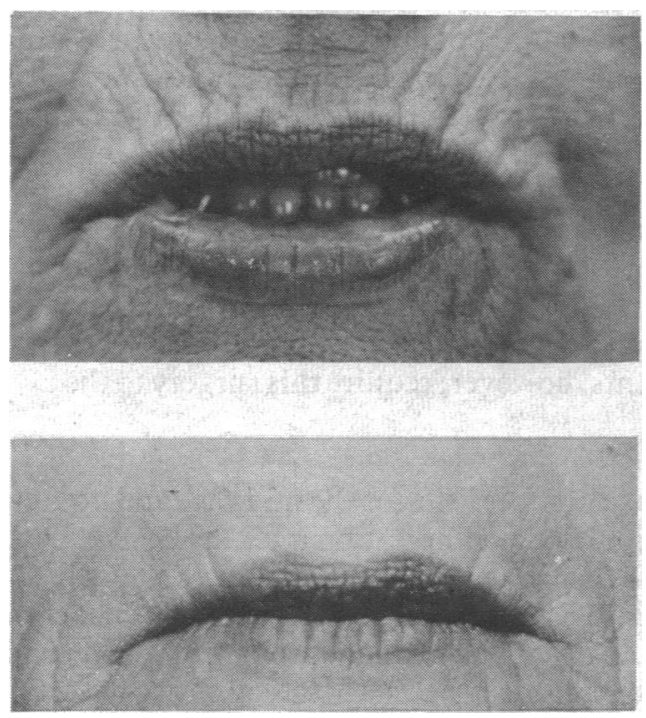

\section{Skin creases}

The more major deep creases, in particular the nasolabial fold and the crows' feet lateral to the eyes are not well managed by the operations described above. The recently introduced, injectible collagen goes some way to overcoming this problem. The bovine dermal collagen is treated by lyophilisation and manufactured in a saline suspension. After a test dose in the forearm to rule out the $2 \%$ of patients who are allergic to bovine protein the skin crease can be raised by a series of injections along it. Over correction is required as initially the saline is absorbed and over six months to a year there is a further relapse depending on the amount of collagen that is removed. Nevertheless, the procedure has a place in the cosmetic armamentarium.

The fine creases that occur particularly around the mouth and eyes are best treated by chemical peel or dermabrasion. In chemical peel a phenol solution is used to produce a chemical burn that causes a loss of the superficial epithelium without producing a full thickness burn. Experience is required in its application to avoid complications. It should be done as an inpatient procedure as initially the pain is severe. In most cases there is a satisfactory smoothing of the fine creases.

The major disadvantage is a change in the pigmentation. Initially there is hyperpigmentation caused by erythema, which matures to produce a hypopigmented scar. There is a danger that when the patient sits in the sun the pale area around the eyes and mouth will produce a panda look. This procedure is also best avoided in dark skinned people because of variable and unpredictable pigment changes.

Phenol can be absorbed through the skin, and extreme caution should be exercised with a total face peel as toxic concentrations of phenol can occur in the blood.

Mr D M Davies, FRCs, is consultant plastic surgeon, West London Plastic Surgery Centre, West Middlesex University Hospital, Isleworth, and consultant plastic surgeon and honorary senior lecturer, Royal Postgraduate Medical School, Hammersmith Hospital, London W12 0HS.
WORDS The word SPHINx brings to the mind's eye of most readers that massive stone sculpture at Gizeh in Egypt, with a man's head and a lion's body. It is known locally as Harmakhis, and it represents royalty. Unconnected directly therewith is the Greek Sphinx. The Greeks named the Egyptian model Sphinx through mistakenly identifying it with a similar monster of their own mythology. The Greek Sphinx had a woman's head, lion's body, serpent's tail, and eagle's wings; she was the issue of a union between the two headed hound Orthros and his mother Echidne, who gave birth also to the Chimaera, a fire breathing goat with a lion's head and serpent's tail; the Hydra, the many headed water serpent; and Cerberus, the three headed hound of hell. One suspects that Echidne was exposed to teratogens, or maybe the Greeks anticipated genetic engineering. Now Hera, wife of top god Zeus, had sent the Sphinx to punish Thebes (never mind what for) by asking every Theban wayfarer a riddle. They all failed to give the right answer and were rewarded by being strangled and then devoured. But along came "Swollen foot" (Oedi/pus) who solved the riddle, whereupon the Sphinx leapt from her perch on the mountain and was dashed to pieces in the valley below.

SPHINX means "strangler" (Gk sphingein, to bind tightly, squeeze, throttle; sphingion, a necklace). The constrictive connotation gives us SPHINCTER, a circular muscle whose contraction partially or completely closes an orifice or the lumen of a tube.

The riddle of the Sphinx gives us SPHINGosine, the base of SPHINGoMYELIN and other SPHINGOLIPIDS, which are found in nervous tissue; also SPHINGOLIPIDOSIS, collective name for a group of hereditary diseases in which an enzyme defect leads to the abnormal storage of sphingolipids in one or more tissues of the body, for example, Tay-Sachs disease. Johann Ludwig Wilhelm Thudichum (1829-1901) left his native Germany in 1854 and settled in England, where in due course he became director of the chemistry and pathology laboratories at St Thomas's Hospital. Describing his work on the chemistry of nervous tissue in 1881 , he wrote: "A body remained insoluble (in ether) ... a and to which, in commemoration of the many enigmas which it presented to the inquirer, I have given the name of Sphingosin."

The Sphinx story is the earliest account of a viva voce examination and the dire consequences of failure. But whereas no examiner has leapt from a window on receiving the right answer, several have nearly died of boredom.-B J FREEDMAN. 